\title{
A Price Too High to Pay
}

\author{
Don Gregory Mascareenhas ${ }^{1}$, Glenn Austin Fernandes², Kristel $^{3}$
}

${ }^{1}$ Assistant Professor, Department of Respiratory Medicine, Father Muller Medial College, Mangalore, Karnataka, India. ${ }^{2}$ Asssitant Professor, Department of General Medicine, Father Muller Medial College, Mangalore, Karnataka, India.

${ }^{3}$ Resident, Department of General Medicine, Father Muller Medial College, Mangalore, Karnataka, India.

\section{ABSTRACT}

India carries the highest Tuberculosis burden in the world. As per the Global TB Report 2017, the estimated incidence of TB in India was approximately 28,00,000 accounting for about a quarter of the world's TB cases. ${ }^{1}$ Uttar Pradesh ranking with the highest number, followed by Maharashtra and Madhya Pradesh and of which death has been reported in $4 \%$ of the detected cases. Pulmonary tuberculosis is diagnosed primarily by chest x-ray findings and sputum smear examination which shows acid fast bacilli. If sputum smear is negative, sputum is subjected to cartridge based nucleic acid amplification test (CBNAAT or GeneXpert) which will detect gene of mycobacterium tuberculosis. If GeneXpert is negative, sputum should be subjected to mycobacterial cultures and efforts should be made to rule out other differential diagnosis like interstitial lung disease, malignancy, non-mycobacterial diseases etc. Computerized tomography (CT) of the chest and subsequently bronchoscopy and cytological/microbiological examination of bronchial washings/lavage will help us in arriving at a diagnosis. Due to the increased prevalence of the disease in our country, many patients who are having radiological opacities on chest $\mathrm{x}$-ray are initiated on antitubercular treatment (ATT) on clinical presumption without microbiological confirmation. We present a case series of patients who were on ATT based on clinical suspicion in primary health centres or peripheral hospitals who consulted our hospital based on non-improvement of symptoms.

\section{KEY WORDS}

TB, Bronchoscopy, CT SCAN, ATT

\section{CASE REPORT}

\section{Case 1}

We present a 25-year-old female who came with complaints of breathlessness and fever since 2 months. Patient had been evaluated at an outside hospital and was started on Anti tubercular Treatment based on Chest X-RAY findings. CT Thorax showed multiple areas of consolidation showing heterogenous enhancement involving right lung with air bronchograms and ground glass opacities in the left lung(Fig 1). Multiple nodular opacities were seen in bilateral lung. Bronchoalveolar lavage came negative for AFB, but atypical cells were noted on cytological examination. CT Guided Biopsy revealed Bronchoalveolar carcinoma. Patient was advised chemotherapy.

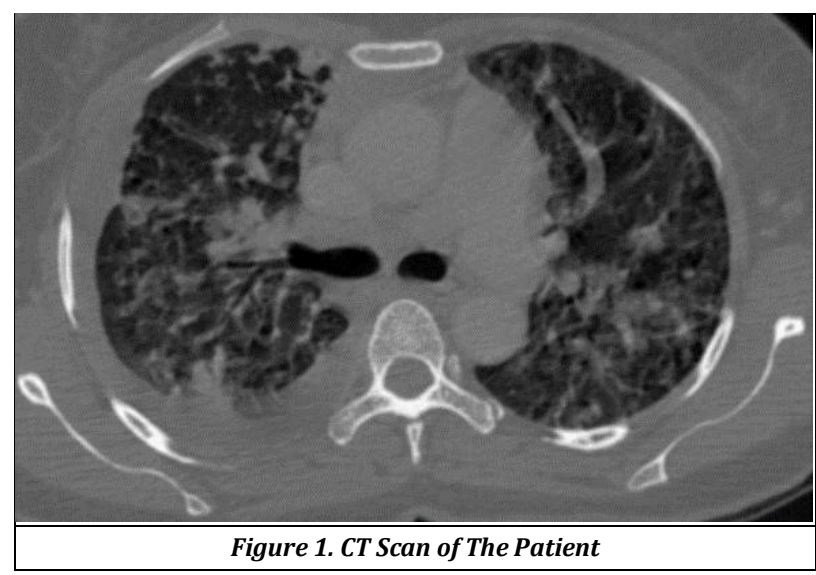

Corresponding Author: Glenn Austin Fernandes, \#203, Mullernest, Valencia. E-mail: glennindia@yahoo.com DOI: $10.14260 /$ jemds $/ 2019 / 549$

Financial or Other Competing Interests: None.

How to Cite This Article: Mascarenhas DG, Fernandes GA, Kristel. A Price too high to Pay. J. Evolution Med. Dent. Sci. 2019;8(31):2523-2525, DOI $10.14260 / \mathrm{jemds} / 2019 / 549$

Submission 26-05-2019,

Peer Review 16-07-2019,

Acceptance 22-07-2019,

Published 05-08-2019. 


\section{Case 2}

A 64-year-old chronic smoker with no other known comorbidities presented with worsening dyspnoea and productive cough since 1 month accompanied with fever since the last 1 week. Patient was being treated with anti-tubercular drugs from outside hospital. Chest X-ray revealed homogenous opacity in left middle and lower zone. CECT Thorax showed a heterogeneously enhancing mass lesion in the left lower lobe likely suggestive of malignancy (fig 2). Bronchoscopy and cytology were positive for atypical cells. Biopsy from the lung mass revealed carcinoma in situ.

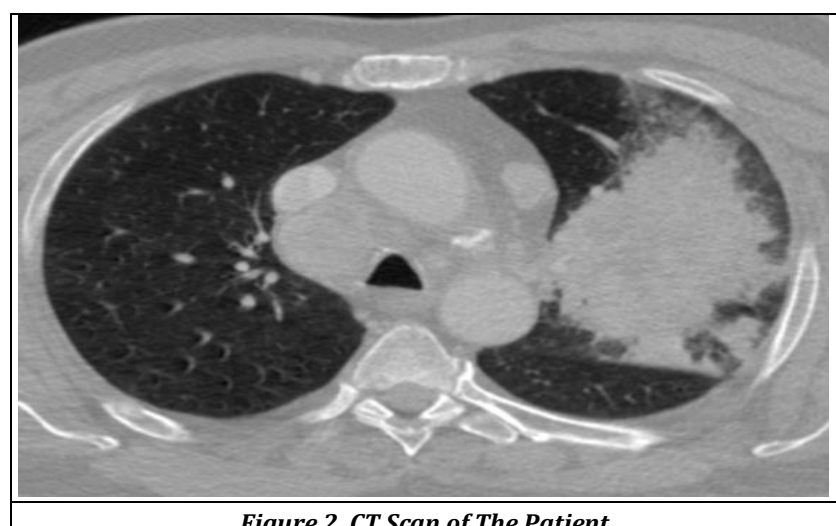

\section{Case 3}

A 60-year-old gentleman diagnosed with left parapneumonic effusion in a peripheral hospital was started on ATT based on lymphocytic predominant cytology. He presented to us with complaints of fever, cough, chest pain and breathlessness since 1 month. At admission, vitals were stable, and patient had pallor with clubbing. Chest X-ray revealed left mid zone and lower zone opacity - likely consolidation with effusion. Pleural fluid was aspirated which was negative for acid fast bacilli (AFB). CT scan was done (fig 3) followed by Bronchoscopy which revealed transbronchial growth in left lower lobe bronchus and biopsy from the lesion revealed moderately differentiated Squamous cell carcinoma.

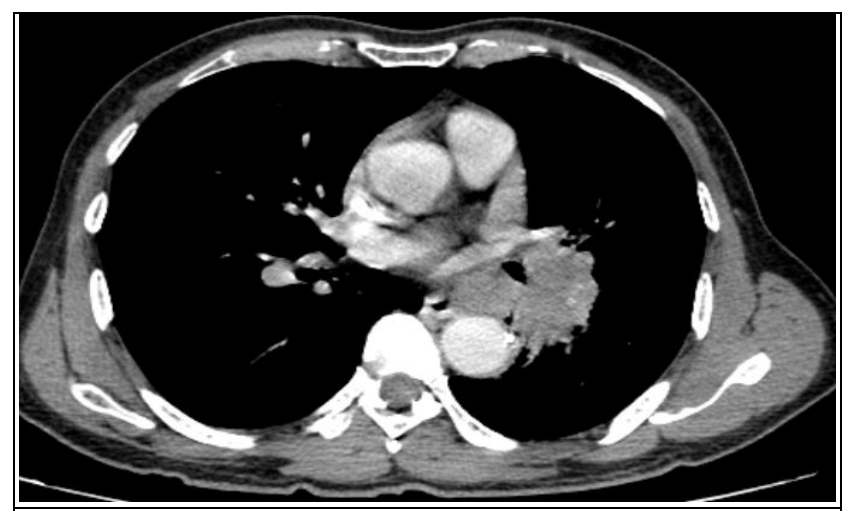

Figure 3. CT Scan of The Patient

\section{Case 4}

A 58-year-old gentleman, who was on ATT for 4 months on presumption of TB based on a left upper zone consolidation came with complaints of haemoptysis since 15 days. Blood picture showed pancytopenia. CT Thorax revealed a welldefined, peripherally enhancing mass lesion in the left upper lobe (Fig 4) and biopsy revealed squamous cell carcinoma (Stage III). Patient was initiated on chemoradiation.

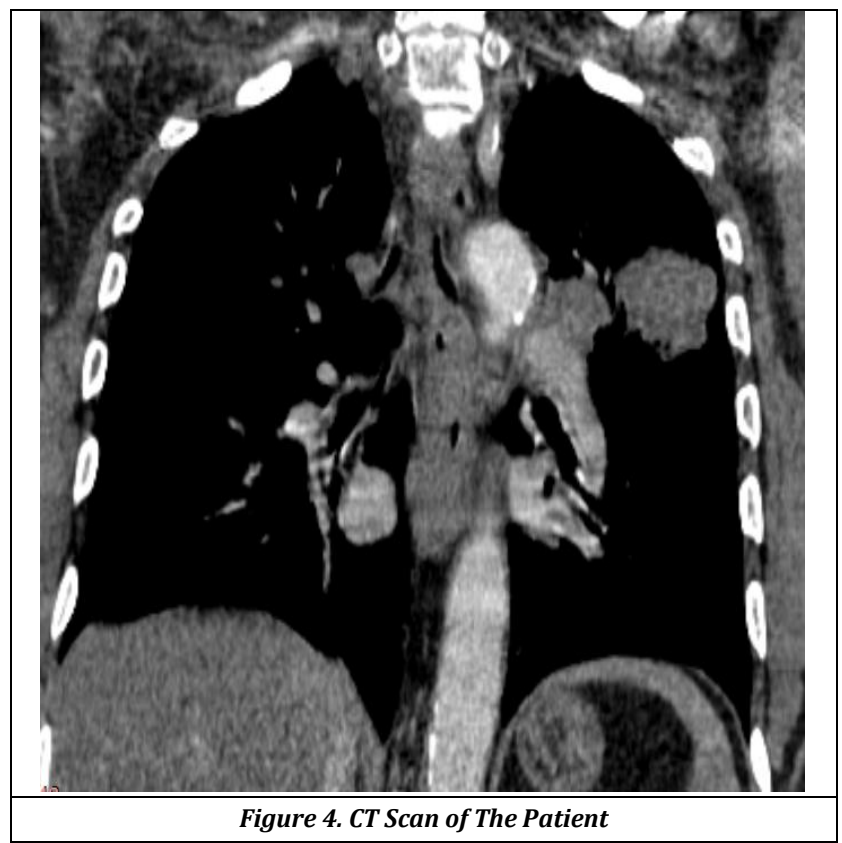

Case 5

A 68 years old lady, who was on treatment for Tuberculosis, presented with cough and dyspnoea. On admission, she was hypoxic. Chest X-ray revealed patchy opacities in bilateral upper and middle zones. CT Thorax revealed patchy ground glass opacities with air bronchograms in bilateral lungs with upper lobe predominance suggestive of cryptogenic organizing pneumonia (Fig 5). She was initiated on Steroids and other supportive measures. Patient improved drastically both clinically and radiologically and was discharged on oral steroids.

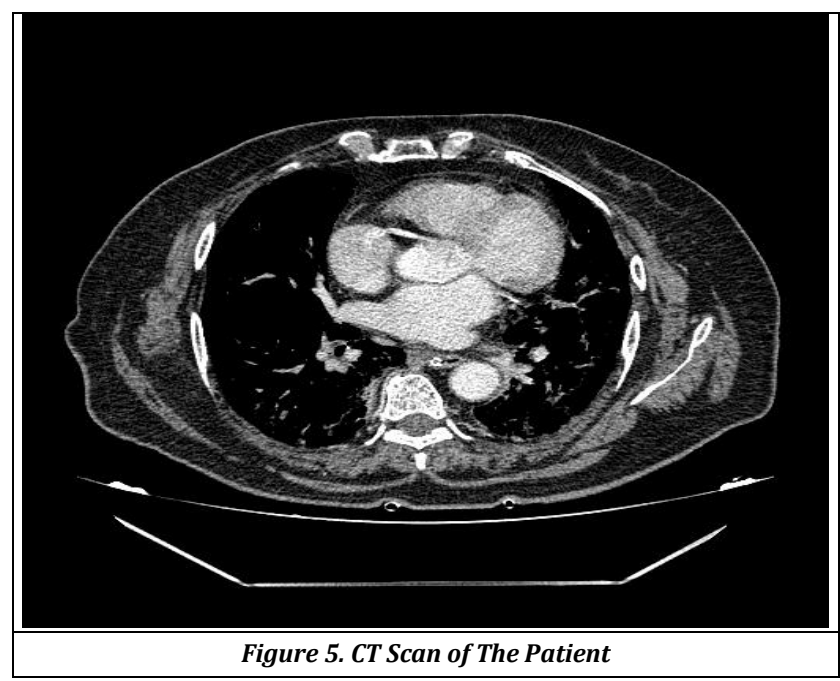

\section{DISCUSSION}

Any lung lesion seen on Chest X-Ray can be either benign or malignant ranging from a benign lung cyst/ ABPA (Allergic Broncho pulmonary Aspergillosis) /Interstitial Lung Disease/ Tuberculosis to a malignancy and hence the initial approach to evaluation should be to establish the nature of the lesion. ${ }^{2}$ Curable disease like ABPA is one of the most underdiagnosed 
condition due to marked radiological similarity to Tuberculosis. ${ }^{3} \mathrm{ABPA}$ has shown an increased predilection in asthmatics and is a progressive condition. If left untreated, chronic disease has shown to cause lung fibrosis as well as cavitary changes henceforth worsening prognosis if diagnoses is delayed. ${ }^{4,5}$ Similarly, Interstitial lung disease is a spectrum of conditions which often gets misdiagnosed or is diagnosed very late causing substantial morbidity to the patient. ${ }^{6}$ The most commonly found subgroups are Idiopathic Pulmonary Fibrosis, Hypersensitivity pneumonitis, those related to connective tissue disorders and those secondary to drug intake. ${ }^{7}$ We as clinicians are also at a disadvantage as only 1 out of every 3 cases have a established aetiology. The mainstay of treatment is steroids and the 5-year survival rate has been found to be $20 \%$ in individuals with IPF whereas $100 \%$ in cryptogenic organising pneumonia. ${ }^{8}$ Lung cancer is one of the most commonly diagnosed malignancy worldwide but Patient with lung cancer being misdiagnosed as tuberculosis is not a rare scenario. A delay in detecting a lung malignancy results drastically varies the prognosis considering the rapidly progressive nature of the disease. Worldwide, approximately 2.09 million cases were reported in 2018 (11.6\% of all cancers) with 1.76 million deaths thereby accounting for $18.4 \%$ of all cancer related deaths. ${ }^{9}$ The doubling time of a lung malignancy vary with the histological type with large cell carcinoma showing the shortest doubling time of 67 days in comparison adenocarcinoma and squamous cell carcinoma which was 221 days and 115 days respectively. ${ }^{10}$ An equally rapid progression of disease was seen in small cell carcinoma with a doubling time of 225 days. Patients diagnosed at Stage 3 or 4 has been found to have a 5 -year survival rate of $<10 \%$ whether it be small cell or non-small cell lung carcinoma. Hence better treatment outcome correlates proportionately to early diagnosis. With the current targeted treatment modalities, against Vascular Endothelial Growth Factor (VEGF), ALK (Anaplastic Lymphocyte Kinase), EGFR (Epithelial Growth Factor Receptor) and so on, disease remission rates have also shown an upward trend.

\section{CONCLUSIONS}

Diagnosis and treatment of tuberculosis has improved a lot in the past decade owing to presence of GeneXpert. However, a lot of cases still get misdiagnosed as TB due to clinical suspicion alone. Patients presenting with cough, fever or suspicion alone. Patients presenting with cough, fever or breathlessness, who have a radiological resemblance to TB should be evaluated further when smear or GeneXpert is negative. When patient presents to a Tertiary Care Centre or when he/she can be referred to a higher centre, care has to be given for further evaluation to reach a diagnosis based on definitive evidence rather than a presumptive conclusion, as a misdiagnosis can cost a patient's life.

\section{REFERENCES}

[1] TB India report 2018. Available at https://tbcindia.gov.in

[2] Del Ciello A, Franchi P, Contegiacomo A, et al. Missed lung cancer: when, where, and why? Diagn Interv Radiol 2017;23(2):118-26.

[3] Bedi RS, Bedi GK. Allergic bronchopulmonary aspergillosis: Indian scenario. Lung India 2007;24(4):156-61.

[4] Shah A, Panjabi C. Allergic bronchopulmonary aspergillosis: a perplexing clinical entity. Allergy Asthma Immunol Res 2016;8(4):282-97.

[5] Shah A. Allergic bronchopulmonary aspergillosis: an Indian perspective. Curr Opin Pulm Med 2007;13(1):7280.

[6] Cosgrove GP, Bianchi P, Danese S, et al. Barriers to timely diagnosis of interstitial lung disease in the real world: the INTENSITY survey. BMC Pulm Med 2018;18(1):9.

[7] Meyer KC. Diagnosis and management of interstitial lung disease. Transl Respir Med 2014;2:4.

[8] Interstitial lung diseases. Available at: https://www.erswhitebook.org/files/public/Chapters/2 2_ILDs.pdf

[9] Bray F, Ferlay J, Soerjomataram I, et al. Global cancer statistics 2018: GLOBOCAN estimates of incidence and mortality worldwide for 36 cancers in 185 countries. CA Cancer J Clin 2018;68(6):394-424.

[10] Arai T, Kuroishi T, Saito Y, et al. Tumor doubling time and prognosis in lung cancer patients: evaluation from chest films and clinical follow-up study. Japanese Lung Cancer Screening Research Group. Jpn J Clin Oncol 1994;24(4):199-204. 